\title{
Product Family Modeling Method for Rapid Response Design
}

\author{
Shouming Hou, a , Lijuan He ${ }^{1, b}$, Hualong Xie ${ }^{2, c}$,Yongxian $\mathrm{Liu}^{2, \mathrm{~d}}$
}

${ }^{1}$ School of Computer Science \&Technology, Henan Polytechnic University ,Jiaozuo, China

${ }^{2}$ School of Mechanical Engineering and Automation, Northeastern University, Shenyang, China

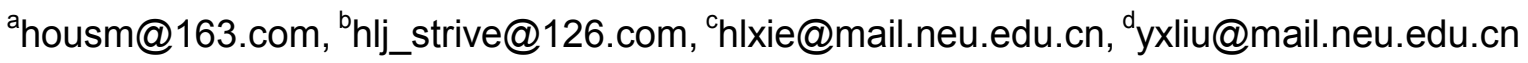

Keywords: Rapid response design, Reusable design resource, Product family, Master model

\begin{abstract}
Design resources reuse is very important to the rapid response design. In this paper, a design resources reusable model based on product family is established to meet the rapid response design. Firstly, parameters transmission structure of product family is constructed based on Generic Bill of Materials (GBOM) and product master model, and then the reused design resource library is established by the instantiation to master model. Finally product configuration management and variant design is realized on the basis of the reused design resource library. In the developing process of the rapid response design system of machine tool component, the Linear Motion (LM) rolling guide variant design subsystem is developed by the application of the above methods to realize the variant design and configuration management of LM rolling guide. The result shows that the method can provide a reliable guarantee for establishment and effective management of corporate design resource base.
\end{abstract}

\section{Introduction}

Design resources reuse is a key technology of rapid response design, collaborative design and virtual design. In the current modeling methods that support product design reuse modeling, reuse of design resources is achieved primarily by the method of configuration management and variant design. The researchers mainly adopt the method of combination of three-dimensional model and process controlling, according to the design requirements of parts or components, a set of design parameters which can control the shape of three-dimensional model and topological relations is established. And relations and constraints among parameters are also established. Finally, certain parameters or partial structure of product is modified through the application of the idea of size variant design; the value of certain parameters and relations is updated so as to drive the model to generate new similar products, which can achieve the purpose of resource reuse [1-3].

However, product information model established by the above method does not cover the whole process of design reuse, moreover, product information, such as variant information, production processing information etc, is not taken into consideration except geometry structure information of products. Thus, it brought a lot of trouble to effective management of design resources.

In this paper, a multi-level, multi-view product design resources reusable model based on the characteristic level is proposed for effectively supporting the design reuse, and variant design modeling method of product family based on the GBOM structure tree and master model technology is discussed and constructed. On this basis, the machine tool parts rapid response design system was developed; it shows the method can meet the requirements of design resources reuse.

\section{Construction of Reusable Design Resource Library}

Reusable Model of Design Resource. Qi Feng, etc. proposed a multi-level, multi-view reusable model of product design resources to meet the requirements of design resource reuse [4]. But it did not consider how to build the design resources reusable model based on 3D design software.

In this paper, the model is expanded to establish the design resource reusable model based on the level of the feature base. Reusable model structure of design resources is shown in Fig. 1. 
In the above model, assembly modeling method of top-down is adopted to make an overall analysis and decomposition to the product model, customer needs information should be made a function mapping on each level and translated into specific product structure information and behaviour information. Firstly, according to enterprise product series, product configuration structure tree is established based on feature base. Then make a parametric modeling of components and the product assembly by means of CAD software. In this way, in the process of product reuse design or variant design, examples of model reference can be generated easily by parameter driven technology of master model, which can improve the response speed greatly.

Process of Establishing Design Resource Library. Design resource library is established on the basis of reusable model of design resource, building process of design resource library is shown in Fig. 2. Design resources make a correlation between product structure feature information and highlevel feature information by means of packaging the reusable model, store the standardized information unit in the design resource library by means of defining model, extracting unit, standardizing unit, the preparation of specifications, classifying and establishing index etc, query, browse, and update the design resource by taking advantage of effective management mechanism in design resource library to provide effective support to reusable design and configuration. Meanwhile, new features, component instances and product examples which are established in each design process can be added to design resource library automatically to meet the needs of design reuse on different level.
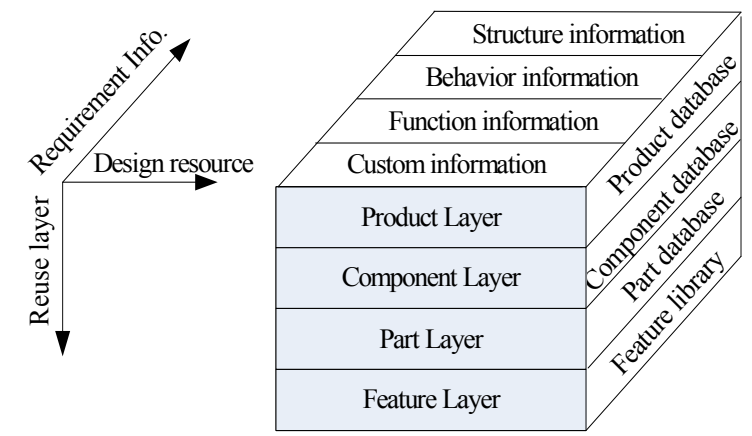

Fig. 1 Reusable model of design resource

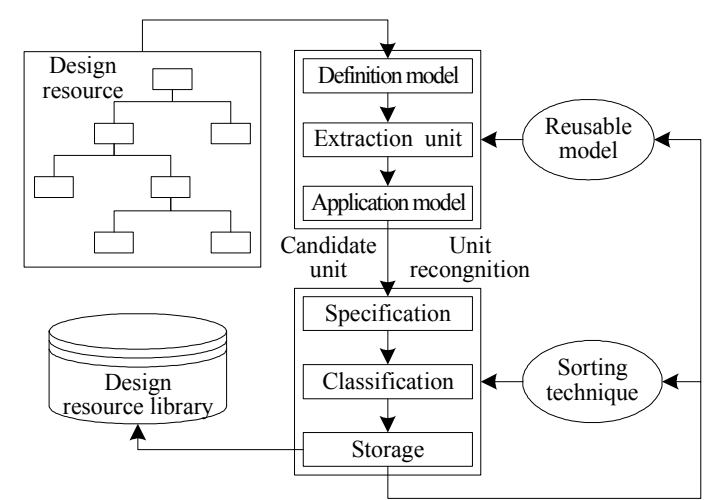

Fig. 2 Process of constructing design resource library

\section{Modeling Method of Reusable Product Family}

In the process of rapid response design to complex product, configuration management and variant design of product family components is the core of design resources reuse. Configuration management and variant design of product family can be achieved by master model technology provided by three-dimensional CAD platform. In this paper, complex product variant master model is divided into four parts: product family GBOM (Generic Bill of Materials) structure [5], product 3D master model, product variant parameters transferring structure and product information master model [6].

GBOM Model of Product Family Based on Polychromatic Sets. GBOM structure of product family is a tree hierarchy structure which is similar to the product BOM structure; it can express the composition of all components included in the product family of the various types of products.

Generic product family GBOM structure is shown in Fig. 3. Class components in Fig. 3 mean the collection of all components which are in the identical node location in product structure. Class parts mean the collection of all parts which are in the identical node location in product structure. In the process of product variant design, any one class components or parts has corresponding variable table which is stored in the SML to represent the changes of node components in order to achieve product configuration design.

In this paper, modeling method based on polychromatic sets theory is adopted to describe tree structure of product family GBOM. 
Components and parts of product are expressed by the nodes of polychromatic graph; their different attributes are expressed by different colors of nodes. The configuration relations of parts can be expressed by different colors painted in edge. Configuration model of product family structure based on polychromatic sets theory can be expressed as follows [7]:

$$
P G=(F(G), P, C, F(P), F(C), R)
$$

In Eq. 1, F(G) represents the overall attribute of product family, $F(G)=\left(F_{1}, F_{2}, F_{3}, \ldots, F\right) . P=$ $\left(\mathrm{P}_{0}, \mathrm{P}_{1}, \mathrm{P}_{2}, \ldots, \mathrm{P}_{\mathrm{m}}\right), \mathrm{P}_{0}$ represents product, $\mathrm{P}_{\mathrm{i}}$ represents components or parts. $\mathrm{F}(\mathrm{P})$ represents different attributes of components or parts, $F(P)=\left(F_{1}, F_{2}, \ldots, F_{1}\right)$, it can represent different meanings according to practical application. $\mathrm{C}=\left(\mathrm{C}_{0(1)}, \mathrm{C}_{0(2)}, \ldots, \mathrm{C}_{\mathrm{j}(\mathrm{k})} \mid 0 \leq \mathrm{j} \leq \mathrm{m}, 0 \leq \mathrm{k} \leq \mathrm{m}\right), \mathrm{C}_{\mathrm{j}(\mathrm{k})}$ represents the vector edge that connects $P_{j}$ and $P_{k} . F(C)=\left(F_{c 1}, F_{c 2}, \ldots, F_{c k}\right)$, it represents different attributes of edge, Fck can denotes different meanings according to practical application.

$\mathrm{R}=\left(\mathrm{R}_{1}, \mathrm{R}_{2}, \ldots, \mathrm{R}_{\mathrm{c}}\right)$, it represents the constraint relations between part nodes. $R_{c}=\left(P_{i j}^{k l}, r e l\right)$, it indicates there is a constraints rel between the end node $\left(\mathrm{P}_{\mathrm{j}}\right)$ of edge $\mathrm{P}_{\mathrm{i}} \mathrm{P}_{\mathrm{j}}$ and the end node $\left(\mathrm{P}_{1}\right)$ of edge $\mathrm{P}_{k} \mathrm{P}_{1}$. General type of relationship rel can include such as dependency, conflicts, exchange, replacement, etc.

Matrix is used to record various attributes set in Polychromatic sets theory. There is a unique matrix to correspond to each constraint in product. All structure of product family can be converted to matrix representation by filling the information matrix, and the matrixes can be expanded to meet the more complex product modeling. This is propitious to the computer programming.

Expression of Product Three-dimensional Master Model. Product 3D master model indicates abstract of components and parts in order to satisfy the functional or assembling requirements of the upper parent-part on all layered structure in enterprise product family model. Product 3D master model has the same characteristics degree of abstraction and follows the rules of similar configuration restraint; it can represent product class, part class, component class on random abstract rank. Product 3D master model can be expressed as follows.

$$
\mathrm{CM}=(\mathrm{ID}, \mathrm{SML}, \mathrm{CP}, \mathrm{Op}, \mathrm{Cons})
$$

In Eq. 2, CM represents the product 3D master model, ID is the identification of CM, SML includes main parameters of $\mathrm{CM} ; \mathrm{CP}=\left(\mathrm{cp}_{1}, \mathrm{cp}_{2} \ldots \mathrm{cp}_{\mathrm{i}}\right)(\mathrm{i} \geq 1)$ is the configuration parameters of $\mathrm{CM}, \mathrm{CP}$ is obtained by mapping of SML's feature set $C=\left(c_{1}, c_{2} \ldots c_{n}\right)(n \geq 1)$. More common situation is to extract features directly from the $\mathrm{C}$ as a configuration parameter. $\mathrm{Op}=\left(\mathrm{op}_{1}, \mathrm{op}_{2} \ldots \mathrm{op}_{\mathrm{m}}\right)(\mathrm{m} \geq 1)$ is a group of operations to $\mathrm{CM}$. Cons $=\left(\operatorname{cons}_{1}, \operatorname{cons}_{2} \ldots\right.$ cons $\left._{\mathrm{i}}\right)(\mathrm{i} \geq 1)$ is a group of constraints set of $\mathrm{CM}$.

Product information master model preserves variant information and production processing information which are outside the geometric information. Different types of documents, such as engineering drawing, NC program, processing technique etc, can be derived by taking advantage of different information master model. Files, databases and PDM systems are frequently adopted to preserve product information, and unified coding system is used to facilitate the database operation.

Structure Model of Variant Parameter Transmission. Product variant parameter transmission structure is established on the basis of product variant parameters constraint relations, it combined with product family GBOM structure, CAD software features and tabular techniques.

In product variant parameter transmission structure, parameters which are passed between upper layer and lower layer include component part geometric variant parameters and product family configuration parameters. Product master models on each levels package their own SML, on different levels of product structure, information stored in tabular is different. In this paper, function characteristic parameter is added to SML through self-definition, and pointer that points to lower component parts or SML is preserved. Based on the pointer, SML_R among component parts master model can be established. The value of feature's global parameter is transited from upper master model to lower master model.

$$
\text { SML_R }=(I D, S M L p, S M L c, \text { Pr })
$$


In Eq. 3, ID is the identification number of associated object, SMLP is identification number of SML of upper master model, SMLc is identification number of lower master model, and $\operatorname{Pr}$ is the transmission relation among parameters of upper master model and lower master model. Through $\mathrm{Pr}$, changes of parameters of any upper master model will lead to changes of corresponding parameters of node in lower master model from up to down.

According to transmission theory of parameters in upper layer and lower layer through product variant parameter, product variant parameter transmission model established is shown in Fig.4. In the model, data file in master model of components is divided into four basic levels: parameter input layer, data processing layer, model-driven layer, parameters output layer. Parameter input layer receives parameters that upper level assembly passed down and parameters in its SML; then after data processing (specific data processing is completed through variant rules), part of the parameter drives components or changes of assembly model, other parameters are separated and transmitted to various subordinates child assemblage and components as configuration parameters.

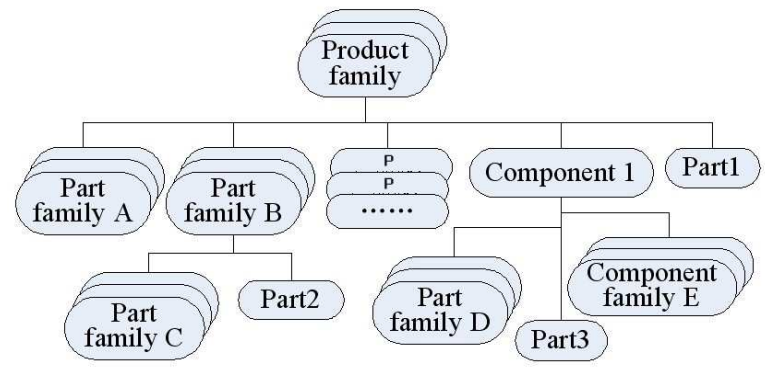

Fig. 3 GBOM structure of product family

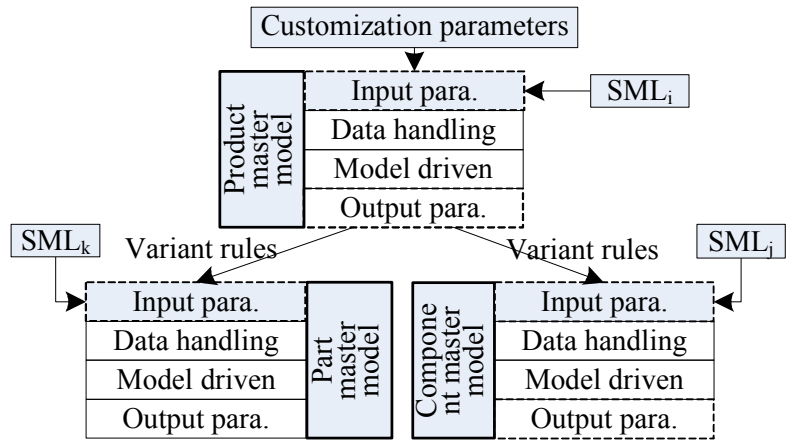

Fig. 4 Parameters transfer model of variant design

Instantiation of Product Master Model. Instantiation process of product master model is shown in Fig. 6. Based on the product information master model and product parameter transmission structure, part of Customization parameters are used to control the configuration of product 3D master model, the other parameters will be passed and acted as the optional parameters of product variant design. during the running process of system, three-dimensional model of customized products can be obtained to meet customers' need through SML of components, variant rules etc which are called by the ID code of products or components; In addition, Customization parameters control structure variant of product family GBOM to generate BOM structure of customized products through variant parameter transmission structure, SML and variant rules; while product information master model gets corresponding data information from customization parameters, 3D model of customized products and BOM structure to form customized product information instance through variant.

\section{Application}

In the rapid response design system of machine tools for mass customization, rolling guide design subsystem was developed to achieve its configuration management and variant design based on UG NX and Teamcenter Engineering developing platform. Fig.5 shows the tree model of product family structure of LM rolling guide.

The master model of guide was established mainly based on UG NX modeling and assembly modeling environment. During the modeling process, UG NX feature modeling, product family modeling technology, electronic forms technology, and UG WAVE technology were used. Fig.6 shows the several 3D models of rolling guide which were generated based on different configuration parameters. 


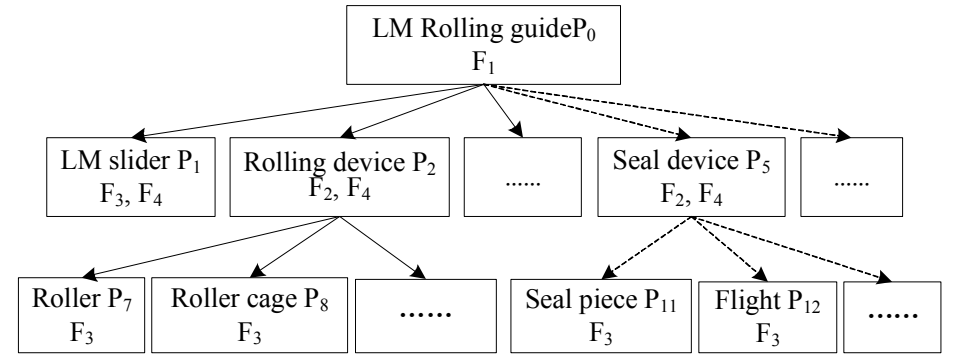

$\mathrm{F}_{1}$ : product item;

$\mathrm{F}_{2}$ : component item;

$\mathrm{F}_{3}$ : part item;

$\mathrm{F}_{4}$ : optional sub-item;

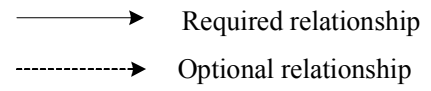

Fig. 5 Product family model of LM rolling guide

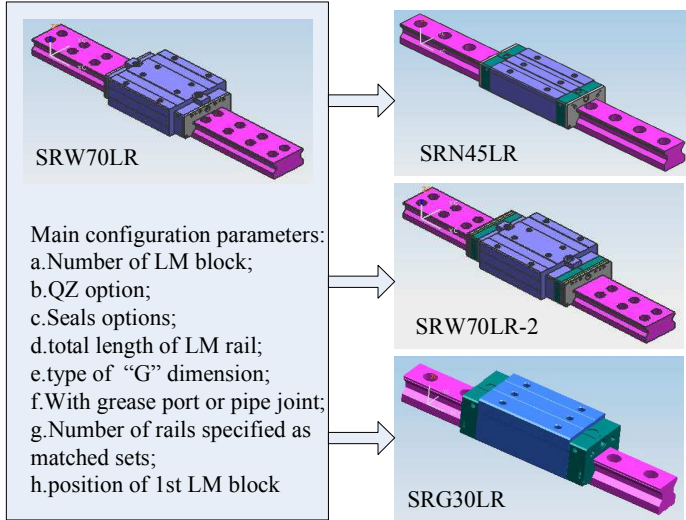

Fig. 6 Configuration management of LM guide

\section{Conclusions}

In this paper, a multi-level, multi-view design model is constructed for reusable resources based on Feature database to meet the design requirements of different levels of reuse, which greatly improve the response speed of product design. In addition, the assembly components product family modeling for design resource reuse is achieved based on the complex products GBOM structure, product 3D master model, product variant parameters transmission structure and product information main model. Lastly, we lay out the variant design and configuration management of LM rolling guide product family by developing a prototype system to prove the feasibility of the proposed method.

\section{References}

[1] J.Y. Zhao, T.-X. Zhong, An Approach to Intelligent Rapid Product Development Based on Product Dynamic Model,Journal of CAD \& CG, 13(2001) 247-252

[2] F. J. Wang, Mills J J, Devarajan V, A conceptual approach managing design resource, Computers in Industry.47(2002)169-183.

[3] Szykman S, Sriram R D,Bochenek C, Design repositories: engineering design's new knowledge base, IEEE Intelligent Systems, 15(2000) 48-55.

[4] Qi Feng, J. R. Tan, S.Y. Zhang, Design Resource Reusable Model and Process for Mass Customization, Computer Integrated Manufacturing Systems, 10(2004) 508-513.

[5] Hegge H M H, Wortmann J C, Generic bill of material: a new product model. International Journal of Production Economics. 23(1991)117-128.

[6] T. Feng, B. Dan, C.L. Lan, Product family structure and configuration management for mass customization, Computer Integrated Manufacturing Systems. 9(2003) 210-213.

[7] M.Q. Yin, G.Y. Zhang, Modeling and configuring product family based on the polychromatic sets, Manufacturing Automation, 30(2008)28-31. 